\title{
BROTAÇÃO E PRODUÇÃO DE MANDIOQUINHA-SALSA ‘AMARELA DE CARANDAÍ', PROVENIENTE DE MUDAS DESINFECTADAS COM ÓLEO DE EUCALIPTO E ENRAIZADAS EM BANDEJAS ( $\left.{ }^{1}\right)$
}

\author{
NÉSTOR ANTONIO HEREDIA ZÁRATE $\left(2^{*}\right)$; KÁTIA REGINA FREITAS SCHWAN-ESTRADA $\left({ }^{3}\right)$; \\ MARIA DO CARMO VIEIRA $\left({ }^{2}\right)$; MARCELO HELMICH $\left({ }^{4}\right)$; RODRIGO VARGAS MACEDO $\left({ }^{5}\right)$; \\ DIEGO MENANI HEID $\left(^{6}\right)$
}

\begin{abstract}
RESUMO
Objetivou-se avaliar a brotação das mudas e a produção de mandioquinha-salsa 'Amarela de Carandaí', provenientes de mudas desinfectadas com óleo de eucalipto e enraizadas em bandejas de isopor. No préenraizamento em bandejas, utilizou-se a matriz experimental Plan Puebla III para combinar concentrações de óleo de eucalipto e tempos de imersão, respectivamente: $0,6 \% / 36 \mathrm{~s} ; 1,4 \% / 36 \mathrm{~s} ; 0,6 \%$ / $84 \mathrm{~s} ; 1,4 \%$ / $84 \mathrm{~s} ; 1,0 \%$ / $60 \mathrm{~s} ; 0,1 \%$ /36 $\mathrm{s} ; 1,9 \% / 84 \mathrm{~s} ; 0,6 \% / 6 \mathrm{~s}$ e $1,4 \% / 114 \mathrm{~s}$, mais uma testemunha $(0,0 \% / 0 \mathrm{~s})$. No campo, utilizaram-se 10 mudas por parcela e seis tratamentos: $0,6 \% \mathrm{~s} ; 36 \mathrm{~s} ; 0,6 \% / 84 \mathrm{~s} ; 1,0 \% / 60 \mathrm{~s} ; 0,1 \% / 36 \mathrm{~s} ; 0,6 \% / 6 \mathrm{~s}$ e $0,0 \% / 0 \mathrm{~s}$. Maiores porcentagens de mudas brotadas e altura de plantas foram obtidas nos tratamentos onde se utilizaram concentrações de óleo de eucalipto menores que $1,0 \%$ e na testemunha. As maiores massas frescas de raízes comerciais, nas colheitas aos 264 dias após o plantio, foram obtidas nos tratamentos 0,6\%/36 s, 0,6\%/84 s e 1,0\%/60 s. Houve queima das folhas provocadas por Alternaria sp e Septoria sp., com maior intensidade aos 111 dias após o plantio, associadas e sem possibilidade de quantificação isoladamente. Concluiu-se que, para se obter maior produção de raízes comerciais, as mudas devem ser desinfectadas em solução aquosa com doses de 0,6\% ou 1,0\% de óleo de eucalipto e imersão em tempos de $36 \mathrm{~s}$ a $84 \mathrm{~s}$, sendo a colheita aos 264 dias após o plantio.
\end{abstract}

Palavras-chave: Arracacia xanthorrhiza, controle alternativo de doenças, pré-enraizamento.

\section{ABSTRACT \\ SPROUTING AND YIELD OF ‘AMARELA DE CARANDAÍ’ PERUVIAN CARROT FROM CUTTINGS DISINFECTED WITH EUCALYPTUS OIL AND ROOTED IN TRAYS}

This work aimed to evaluate the sprouting of cuttings and yield of 'Amarela de Carandaí peruvian carrot plants from cuttings which were disinfected with eucalyptus oil and rooted in polyethylene trays. In pre-rooting on trays, the Plan Puebla III experimental matrix was used to combine doses of eucalyptus oil and immersion periods, respectively: $0.6 \% / 36 \mathrm{~s} ; 1.4 \% / 36 \mathrm{~s} ; 0.6 \% / 84 \mathrm{~s} ; 1.4 \% / 84 \mathrm{~s} ; 1.0 \% / 60 \mathrm{~s} ; 0.1 \% / 36 \mathrm{~s} ; 1.9 \% / 84 \mathrm{~s} ; 0.6 \% / 6 \mathrm{~s}$ and $1.4 \% / 114 \mathrm{~s}$, added a control $(0.0 \% / 0 \mathrm{~s})$. At field, ten cuttings per plot and six treatments were used: $0.6 \% / 36 \mathrm{~s} ; 0.6 \% / 84 \mathrm{~s} ; 1.0 \% / 60$ $\mathrm{s} ; 0.1 \% / 36 \mathrm{~s} ; 0.6 \% / 6 \mathrm{~s}$ and $0.0 \% / 0 \mathrm{~s}$. Highest percentages of sprouted cuttings and height of plants were obtained in treatments with doses of eucalyptus oil smaller than $1.0 \%$ and the control. The highest fresh weight of commercial roots, which were harvested at 264 days after planting, were obtained in $0.6 \% / 36 \mathrm{~s}, 0.6 \% / 84 \mathrm{~s}$ and $1.0 \% / 60 \mathrm{~s}$ treatments. There was burning of leaves caused by Alternaria sp. and Septoria sp., with more intensity on 111 days after planting, which showed theirselves associated and without possibility of isolate quantification. It was concluded that, to achieve higher yield of commercial roots, it must disinfect the cuttings with aqueous solution with doses of $0.6 \%$ or $1.0 \%$ of eucalyptus oil and immersion for periods of $36 \mathrm{~s}$ to $84 \mathrm{~s}$, with harvest at 264 days after planting.

Key words: Arracacia xanthorrhiza, alternative control of diseases, pre-rooting.

(1) Recebido para publicação em 24 de abril de 2009 e aceito em 10 de maio de 2010.

(2) UFGD-FCA, Caixa Postal 533, 79804-970 Dourados (MS). Bolsista de Produtividade em Pesquisa do CNPq. FCA/UFGD Pósdoutorando na UEM. E-mail: nahz@terra.com.br $\left(^{*}\right)$ Autor correspondente.

(3) Universidade Estadual de Maringá, UEM-CCA-Departamento de Agronomia, Av. Colombo, 5790, 87020-900 Maringá (PR). E-mail: krfsestrada@uem.br

(4) Mestrando em Agronomia na UFGD, UFGD-FCA, Caixa Postal 533, 79804-970 Dourados (MS). E-mail: marcelohelmich@hotmail.com

(5) Bolsista IC/CNPq, Rua Quintino Bocaiúva 717, ap. 302. 79800-000 Dourados (MS). E-mail: rodrigo_fbpa@hotmail.com

$\left.{ }^{(}\right)$Bolsista PIBIC do CNPq/UFGD, Rua Francisco Luiz Viegas 1695, 79831-390 Dourados (MS). E-mail: diegoheid@hotmail.com 
Amultiplicação da mandioquinha-salsa (Arracacia xanthorrhiza Bancroft), para fins comerciais é feita, exclusivamente, por mudas obtidas dos rebentos que se formam na coroa, os quais variam em comprimento e diâmetro em função do clone e da idade da planta. Comercialmente, emprega-se na propagação apenas a porção apical do rebento $(2,5$ a $3,0 \mathrm{~cm})$, o qual é retirado de plantas maduras, com cerca de 8-12 meses de idade (LeBLANC et al., 2008). A capacidade de a planta produzir bem depende, principalmente, da qualidade do material de plantio que determina diferenças na velocidade de enraizamento, crescimento e, consequentemente, na produção e duração do ciclo vegetativo (HEREDIA ZÁrATE et al., 2009).

LEBLANC et al. (2008) citam que vários autores propuseram o pré-enraizamento das mudas em canteiros como forma de minimizar riscos de perdas de estande no campo e de possibilitar a seleção de mudas a serem transplantadas. Todavia, resultados conflitantes, quanto ao pré-enraizamento da mandioquinha, têm sido observados nas referências consultadas. Assim, a técnica de pré-enraizamento necessita de avaliações em diferentes locais e épocas de cultivo, sobretudo para novos clones de mandioquinha.

Sobre o tratamento das mudas de mandioquinhasalsa, há poucas informações, embora um dos problemas de cultivo mais frequentes seja o baixo estande final, em relação ao número de mudas plantadas, causado, muitas vezes, pelo ataque de patógenos comuns em plantios comerciais, como Rhizopus sp. e Erwinia sp. (BRUNE et al., 1996). O tratamento fitossanitário dos rebentos, após o destaque da planta-mãe, é prática indispensável. Recomenda-se sua imersão por 5 a 10 minutos em solução de água sanitária comercial (teor médio de 2,2\% de hipoclorito de sódio), na proporção de $1 \mathrm{~L}$ de água sanitária para $9 \mathrm{~L}$ de água, seguida de secagem à sombra (MADEIRA e SouZA, 2008). Trabalhos desenvolvidos com extrato bruto ou com óleo essencial, obtido a partir de plantas medicinais, têm indicado o potencial delas no controle de fitopatógenos, tanto por sua ação fungitóxica direta, inibindo o crescimento micelial e a germinação de esporos, quanto pela indução de fitoalexinas (SCHWAN-EsTRADA et al., 2000).

Objetivou-se com o trabalho avaliar a brotação de mudas e a produção de plantas de mandioquinhasalsa 'Amarela de Carandaí', proveniente de mudas desinfectadas com óleo de eucalipto (Eucaliptus globulus Labill) e enraizadas em bandejas de isopor.

O trabalho foi desenvolvido em Dourados (MT), no período de 5/ 2008 a 1/ 2009 e composto de duas fases: pré-enraizamento em bandejas e cultivo no campo com as mudas sobreviventes, em solo do tipo Latossolo Vermelho Distroférrico, textura muito argilosa. A análise feita no Laboratório de Solos do FCA - UFGD resultou em: 6,4 de
pH em $\mathrm{H}_{2} \mathrm{O} ; 28,5 \mathrm{~g} \mathrm{dm}^{-3}$ de M.O., $35 \mathrm{mg} \mathrm{dm}^{-3}$ de Pe 8,7;47,3 e 25,6 $\mathrm{mmol}_{\mathrm{c}} \mathrm{dm}^{-3} \mathrm{de} \mathrm{K}$, Ca e $\mathrm{Mg}$, respectivamente, CTC de $126,6 \mathrm{mmol}_{\mathrm{c}} \mathrm{dm}^{-3}$, SB de $81,6 \mathrm{mmol}_{\mathrm{c}} \mathrm{dm}^{-3} \mathrm{e} \mathrm{V}$ de $64 \%$.

O pré-enraizamento em bandejas foi iniciado em 1. ${ }^{\circ} / 5 / 2008$, com a desinfecção das mudas com óleo de eucalipto. Os tratamentos foram $0,6 \% / 36 \mathrm{~s} ; 1,4 \% / 36 \mathrm{~s}$; $0,6 \% / 84 \mathrm{~s} ; 1,4 \% / 84 \mathrm{~s} ; 1,0 \% / 60 \mathrm{~s} ; 0,1 \% / 36 \mathrm{~s} ; 1,9 \% / 84 \mathrm{~s} ;$ $0,6 \% / 6$ s e $1,4 \% / 114 \mathrm{~s}$, determinados utilizando-se a matriz experimental Plan Puebla III (TuRRENT e LAIRD, 1975), além de uma testemunha (sem desinfecção com óleo). O delineamento experimental utilizado foi blocos casualizados, sendo dez tratamentos e quatro repetições. Cada unidade experimental foi formada por 18 mudas.

No dia anterior ao plantio, as mudas foram desinfectadas, de acordo com os arranjos dos fatores em estudo. $\mathrm{O}$ óleo foi misturado com água, formando solução molar e para melhorar a aderência do óleo nas mudas utilizou-se o espalhante-adesivo Cittowett ${ }^{\circledR}$, na dose correspondente a $40 \mathrm{~mL}$ para 100 litros de água. $\mathrm{O}$ pré-enraizamento foi feito em bandejas de poliestireno de 72 células, preenchidas com substrato Plantmax $\mathrm{HA}^{\circledR}$ para hortaliças. No dia do plantio, os rebentos foram cortados horizontalmente na parte basal e enterrados verticalmente dentro de cada célula, com os ápices para cima e deixando-se ao descoberto $\pm 1,0$ $\mathrm{cm}$ de pseudocaule. Foram feitas irrigações diárias com regador e avaliações da brotação das mudas e incidência de doenças. Quando as mudas maiores alcançaram mais de $10 \mathrm{~cm}$ de altura, aos 42 dias após o plantio-DAP, considerou-se que essas estavam em fase de transplante e determinaram-se as porcentagens de mudas brotadas, altura das plantas, diâmetro do pseudocaule e número de folhas. Os dados foram submetidos à análise de variância e, quando se detectou significância pelo teste $\mathrm{F}$, as médias foram comparadas pelo teste de Scott-Knott, a $5 \%$ de probabilidade.

Com as mudas pré-enraizadas nas bandejas implantou-se um experimento no campo, respeitando os tratamentos utilizados na fase de propagação e que permitisse utilizar 10 plantas por unidade experimental. Em consequência, os tratamentos foram de 0,6\%/36 s; $0,6 \% / 84 \mathrm{~s} ; 1,0 \% / 60 \mathrm{~s} ; 0,1 \% / 36 \mathrm{~s} ; 0,6 \% / 6 \mathrm{~s}$ e a testemunha $(0,0 \% / 0 \mathrm{~s})$, resultado das combinações de doses de óleo de eucalipto e tempos de imersão respectivamente. $\mathrm{O}$ delineamento experimental foi o de blocos casualizados, com seis tratamentos e quatro repetições. Cada parcela foi formada por um canteiro de $1,50 \mathrm{~m}^{2}(1,50 \mathrm{~m}$ de largura e 1,00 m de comprimento) e área útil de $1,0 \mathrm{~m}^{2}$ (1,00 m de largura $\times 1,00 \mathrm{~m}$ de comprimento), com 10 plantas, arranjadas em fileira dupla, com espaçamentos de $50 \mathrm{~cm}$ entre fileiras simples e $20 \mathrm{~cm}$ entre plantas, perfazendo a população de 66.000 plantas ha-1.

O terreno foi preparado uma semana antes do plantio, com uma aração e uma gradagem e, 
posteriormente, foram levantados os canteiros com rotoencanteirador. As plantas foram transplantadas em $13 / 6 / 2008$, quando as maiores estavam com mais de 10 $\mathrm{cm}$ de altura. Foram abertos sulcos de plantio de $5 \mathrm{~cm}$ de largura e $5 \mathrm{~cm}$ de profundidade, onde foram colocadas as mudas. As irrigações foram feitas utilizando o sistema de aspersão, com turno de rega diário, até que as plantas tiveram em torno de $20 \mathrm{~cm}$ de altura, e, posteriormente, a cada dois dias. Não foram feitas adubação e calagem para corrigir o solo. As capinas foram feitas com enxada, entre os canteiros, e manualmente, dentro dos canteiros.

Durante o ciclo de cultivo, avaliaram-se o pegamento das mudas, aos $86 \mathrm{DAP}$; a sobrevivência das plantas, aos $224 \mathrm{DAP}$ e os sintomas de doenças na parte aérea das plantas, aos 111, 146 e 183 DAP, avaliando-se a severidade e a incidência. No solo, avaliou-se apenas a incidência, aos 214 DAP. A severidade das doenças foi determinada visualmente, utilizando-se escala de 0,00 (sem sintoma de infecção) a 5,00 (planta morta).

Foram realizadas duas colheitas, sendo a primeira aos 218 DAP e a segunda aos 264 DAP, quando as plantas tinham como indicativo do ponto de colheita o amarelecimento e secamento de mais de $50 \%$ (HEREDIA ZÁrATE et al., 2008b) e de 70\%, respectivamente, das folhas externas das plantas. Nas colheitas foram avaliadas as produções de massas frescas de folhas, rebentos, coroas e raízes comerciais (maiores que $30 \mathrm{~g}$ ) e não-comerciais (menores que $30 \mathrm{~g}$ ). Os dados foram submetidos à análise de variância e, quando se detectou significância pelo teste $\mathrm{F}$, as médias foram comparadas pelo teste de Scott-Knott, a 5\% de probabilidade.

A brotação das mudas, a altura das plantas, o diâmetro do coleto do pseudocaule e o número de folhas por planta aos 42 dias após o plantio foram influenciados significativamente pelos tratamentos (Tabela 1). As maiores porcentagens de mudas brotadas e as maiores alturas das plantas ocorreram nas mudas que foram desinfectadas em solução aquosa com óleo de eucalipto, em concentrações menores que $1,0 \%$, sem diferir da testemunha. Numericamente, a melhor combinação foi de $0,1 \% / 36$ s e a pior foi de 1,9\%/84 s. De acordo com a porcentagem de brotação, diâmetro do coleto e número de folhas deve-se evitar concentrações de óleo de 1,9\% e tempo de imersão de $84 \mathrm{~s}$.

Os 42 dias de permanência das mudas nas bandejas e o número de folhas por planta $(1,52$ e 2,03, nos tratamentos $1,9 \% / 84$ s e $0,6 \% / 36$ s respectivamente) obtidas na primeira fase do trabalho foram inferiores aos valores citados por LeBLANC et al. (2008) que, trabalhando com a mandioquinha-salsa Roxa de Viçosa, seleção dentro da 'Amarela de Carandaí', observaram que as plantas alcançaram o ponto de transplante entre 45 e $60 \mathrm{DAP}$, com 4-5 folhas. Essas diferenças podem ser devidasàs diferenças genéticas entre os clones, às condições de cultivo: bandeja e canteiro e as condições ambiente dos locais de propagação, ou seja, em Dourados (MS) e Viçosa (MG).

A sobrevivência das plantas no campo não mostrou efeito significativo dos tratamentos, apesar das densidades de plantas terem ficado entre 47.850 e 66.000 plantas ha ${ }^{-1}(\mathrm{C} . \mathrm{V} .=14,29 \%)$ e entre 47.500 e 62.700 plantas ha $^{-1}(\mathrm{C} . \mathrm{V} .=14,48 \%)$, respectivamente, nos tratamentos testemunha e 0,6\%/84s, tanto na colheita aos 218 DAP quanto aos 264 DAP. Esses resultados revelam que os sistemas vegetais têm mecanismos de autorregulação, com base na capacidade de adaptação do organismo individual e das populações ou no equilíbrio das relações de interferência, como competição por nutrientes, água e outros (HEREDIA ZÁrATE et al., 2008a).

$\mathrm{Na}$ colheita aos 218 DAP, houve influência significativa dos tratamentos sobre as produções de

Tabela 1. Brotação das mudas e altura, diâmetro do coleto e número de folhas de plantas de mandioquinha-salsa 'Amarela de Carandai', propagadas em bandejas com mudas desinfectadas com diferentes doses de óleo de eucalipto e tempos de imersão das mudas

\begin{tabular}{lcccc}
\hline Tratamento $^{(1)}$ & Brotação & Altura & Diâmetro do coleto & Folhas \\
\hline & $\%$ & $\mathrm{~cm}$ & $\mathrm{~mm} .^{\circ}$ & $2,03 \mathrm{a}$ \\
$0,6 / 36$ & $63,89 \mathrm{a}$ & $7,64 \mathrm{a}$ & $2,02 \mathrm{a}$ & $1,99 \mathrm{a}$ \\
$1,4 / 36$ & $27,78 \mathrm{c}$ & $6,74 \mathrm{~b}$ & $2,14 \mathrm{a}$ & $1,87 \mathrm{a}$ \\
$0,6 / 84$ & $61,11 \mathrm{a}$ & $7,90 \mathrm{a}$ & $1,85 \mathrm{~b}$ & $1,75 \mathrm{~b}$ \\
$1,4 / 84$ & $27,78 \mathrm{c}$ & $6,31 \mathrm{~b}$ & $1,94 \mathrm{a}$ & $1,65 \mathrm{~b}$ \\
$1,0 / 60$ & $45,84 \mathrm{~b}$ & $6,84 \mathrm{~b}$ & $2,05 \mathrm{a}$ & $2,01 \mathrm{a}$ \\
$0,1 / 36$ & $73,61 \mathrm{a}$ & $8,14 \mathrm{a}$ & $1,99 \mathrm{a}$ & $1,52 \mathrm{~b}$ \\
$1,9 / 84$ & $18,06 \mathrm{c}$ & $6,60 \mathrm{~b}$ & $1,65 \mathrm{c}$ & $2,02 \mathrm{a}$ \\
$0,6 / 6$ & $68,06 \mathrm{a}$ & $7,92 \mathrm{a}$ & $2,02 \mathrm{a}$ & $1,77 \mathrm{~b}$ \\
$1,4 / 114$ & $34,72 \mathrm{c}$ & $6,92 \mathrm{~b}$ & $1,79 \mathrm{~b}$ & $1,92 \mathrm{a}$ \\
$0,0 / 0$ & $63,69 \mathrm{a}$ & $7,59 \mathrm{a}$ & $2,11 \mathrm{a}$ & 9,33 \\
C.V. $(\%)$ & 18,49 & 11,31 & 5,48 & \\
\hline
\end{tabular}

$\left({ }^{1}\right)$ Doses de óleo de eucalipto, em porcentagem na solução/tempo de imersão das mudas, em segundos.

Médias seguidas pelas mesmas letras nas colunas não diferem entre si, pelo teste de Scott-Knott, a 5\% de probabilidade. 
massas frescas de coroa e de raiz não-comercial em relação à testemunha (Tabela 2). Na colheita aos 264 dias, as influências significativas foram observadas nas massas frescas de rebento, raiz comercial e raiz nãocomercial (Tabela 2).

Numericamente, as maiores massas frescas de raízes comerciais, nas colheitas aos 218 DAP e aos 264 $\mathrm{DAP}$, respectivamente, foram provenientes das plantas dos tratamentos $0,6 \% / 84 \mathrm{~s}$ e $1,0 \%$ e $60 \mathrm{~s}$ e as menores massas frescas foram dos tratamentos-testemunha e $0,1 \% / 36$ s respectivamente. No aumento de massa das raízes comerciais obtido na colheita aos 264 DAP, em relação às dos $218 \mathrm{DAP}$, observa-se relação inversa com a massa de raízes não-comerciais, confirmando o relatado por Heredia ZÁrate et al. (2009) de que as plantas podem proporcionarar taxas variáveis de crescimento e morfologia bem características, com modificações no fim do ciclo vegetativo.

A maior produção de raízes comerciais obtidas neste experimento $\left(9,60 \mathrm{t} \mathrm{ha}^{-1}\right)$, na colheita aos 264 DAP, foi superior à maior produção $\left(8,04 \mathrm{t} \mathrm{ha}^{-1}\right)$ obtida por HEREDIA ZÁrate et al. (2008b), que trabalhou com consórcio de mandioquinha-salsa com cebolinha (Allium fistulosum L.) e salsa [Petroselinum crispum (Mill.)], mas foi inferior à maior produção $\left(11,61 \mathrm{t} \mathrm{ha}^{-1}\right)$ obtida por Heredia Zárate et al. (2009), que estudou tamanho de mudas e número de plantas no canteiro.

As maiores produções de massas frescas das plantas originadas de mudas desinfectadas com as maiores doses de óleo de eucalipto $(0,6 \%$ e $1,0 \%)$ e as menores produções com as mudas tratadas com as doses mínimas $(0,1 \%$ e $0,0 \%)$, numericamente na colheita aos 218 DAP e estatisticamente aos 264 DAP (exceto 0,6\% e 6 s), confirmam o relatado por KuHN (2007) quando cita que, muitos trabalhos foram desenvolvidos e a eficiência da indução de resistência foi comprovada; no entanto, poucos pesquisadores se preocuparam com a produtividade das culturas em meio a esse fenômeno.
Foram observados sintomas de queima das folhas, com avaliações aos 111, 146 e 183 DAP e de podridões das coroas e dos rebentos aos 214 DAP. Os agentes patogênicos responsáveis pela queima das folhas foram Alternaria sp e Septoria sp., associados e sem possibilidade de quantificação isoladamente. A severidade das infecções foi dependente da interação tratamento das mudas e da época de avaliação (Tabela 3). A maior severidade da doença (dos fungos foliares) foi observada nas plantas originadas de mudas sem desinfecção (Testemunha) e a menor severidade nas plantas originadas de mudas tratadas com $0,6 \%$ de óleo, com mudanças mínimas de acordo ao tempo de imersão. Em relação às podridões, Sclerotium sp. aos 214 DAP, provocou a morte de uma planta da testemunha.

A menor severidade fitopatogênica observada nas plantas originadas de mudas desinfectadas com oóleo de eucalipto sugere que a provável proteção conferida pelo óleo foi capaz da indução de resistência (ou indução de proteção, imunidade adquirida ou resistência sistêmica adquirida) que envolve a ativação de mecanismos de defesa latentes, existentes nas plantas em resposta ao tratamento com agentes bióticos ou abióticos (SCHWANEstRADA et al., 2000).

Os sintomas de queima nas folhas foram observados com maior severidade e incidência, respectivamente, nas épocas em que a temperatura e a pluviosidade aumentaram. Em relação à queima nas folhas, foram observadas duas épocas críticas. A primeira foi a partir do segundo decêndio de agosto $(\approx$ 110 DAP), quando as temperaturas diurnas e noturnas aumentaram e ficaram em torno de $30{ }^{\circ} \mathrm{C}$ e $15{ }^{\circ} \mathrm{C}$ respectivamente, e a umidade do ambiente aumentou devido às altas precipitações pluviais ocorridas no período, em torno de $100 \mathrm{~mm}$. A segunda época foi no início de outubro $(\approx 180 \mathrm{DAP})$, quando a temperatura e as precipitações pluviais iniciaram novo ciclo de aumento. Quanto às podridões, a única planta morta

Tabela 2. Massas frescas de folhas, rebentos, coroas, raízes comerciais e raízes não-comerciais de plantas de mandioquinha-salsa

'Amarela de Carandai', propagadas com mudas desinfectadas com diferentes doses de óleo de eucalipto e tempos de imersão, aos 218 e 264 dias após o plantio

\begin{tabular}{|c|c|c|c|c|c|c|c|c|c|c|}
\hline \multirow{3}{*}{ Tratamento $^{(1)}$} & \multicolumn{10}{|c|}{ Produção de massa fresca $\left(\mathrm{t} \mathrm{ha}^{-1}\right)$} \\
\hline & \multicolumn{2}{|c|}{ Folhas } & \multicolumn{2}{|c|}{ Rebento } & \multicolumn{2}{|c|}{ Coroa } & \multicolumn{2}{|c|}{ Raiz comercial } & \multicolumn{2}{|c|}{ Raiz não-comercial } \\
\hline & 218 & 264 & 218 & 264 & 218 & 264 & 218 & 264 & 218 & 264 \\
\hline $0,6 \%$ e $36 \mathrm{~s}$ & $4,89 \mathrm{a}$ & $4,37 \mathrm{a}$ & $2,52 \mathrm{a}$ & $5,68 \mathrm{a}$ & $3,74 \mathrm{a}$ & $3,67 \mathrm{a}$ & $4,83 \mathrm{a}$ & $8,27 \mathrm{a}$ & $2,69 \mathrm{~b}$ & $2,60 \mathrm{a}$ \\
\hline $0,6 \%$ e $84 \mathrm{~s}$ & $4,34 \mathrm{a}$ & $4,43 \mathrm{a}$ & $3,24 \mathrm{a}$ & $5,39 \mathrm{a}$ & $3,86 \mathrm{a}$ & $4,42 \mathrm{a}$ & $5,36 \mathrm{a}$ & $8,50 \mathrm{a}$ & $4,22 \mathrm{a}$ & $2,56 \mathrm{a}$ \\
\hline $1,0 \%$ e $60 \mathrm{~s}$ & $4,22 \mathrm{a}$ & $2,90 \mathrm{a}$ & $2,51 \mathrm{a}$ & $4,33 \mathrm{~b}$ & $3,61 \mathrm{a}$ & $3,59 \mathrm{a}$ & $4,60 \mathrm{a}$ & $9,60 \mathrm{a}$ & $3,65 \mathrm{a}$ & $2,70 \mathrm{a}$ \\
\hline $0,1 \%$ e $36 \mathrm{~s}$ & $4,18 \mathrm{a}$ & $2,88 \mathrm{a}$ & $2,77 \mathrm{a}$ & $4,85 \mathrm{~b}$ & 3,66 a & $4,14 \mathrm{a}$ & $3,62 \mathrm{a}$ & $5,41 \mathrm{~b}$ & $2,42 \mathrm{~b}$ & $2,45 \mathrm{a}$ \\
\hline $0,6 \%$ e $6 \mathrm{~s}$ & $4,05 \mathrm{a}$ & $3,75 \mathrm{a}$ & $2,66 \mathrm{a}$ & $5,97 \mathrm{a}$ & $3,26 \mathrm{~b}$ & $4,58 \mathrm{a}$ & $5,22 \mathrm{a}$ & $7,25 \mathrm{~b}$ & $3,20 \mathrm{a}$ & $2,92 \mathrm{a}$ \\
\hline $0,0 \%$ e $0 \mathrm{~s}$ & $3,84 \mathrm{a}$ & $3,04 \mathrm{a}$ & $2,50 \mathrm{a}$ & $4,27 \mathrm{~b}$ & $2,75 \mathrm{~b}$ & $3,64 \mathrm{a}$ & $3,47 \mathrm{a}$ & $7,76 \mathrm{~b}$ & $2,21 \mathrm{~b}$ & $1,26 \mathrm{~b}$ \\
\hline C.V. (\%) & 33,78 & 28,99 & 37,59 & 16,03 & 13,10 & 17,26 & 27,06 & 18,40 & 16,58 & 12,45 \\
\hline
\end{tabular}

$\left({ }^{1}\right)$ Doses de óleo de eucalipto, em porcentagem na solução/tempo de imersão das mudas, em segundos.

Médias seguidas pelas mesmas letras nas colunas não diferem entre si, pelo teste de Scott-Knott, a 5\% de probabilidade 
Tabela 3 - Severidade da infecção com doenças foliares de plantas de mandioquinha-salsa 'Amarela de Carandai', propagadas com mudas desinfectadas com diferentes doses de óleo de eucalipto e tempos de imersão, em três épocas de avaliação após o plantio

\begin{tabular}{lcccc}
\hline & Tratamento $^{(1)}$ & \multicolumn{3}{c}{ Época de avaliação (Dias após o plantio) } \\
\hline Dose de óleo & Tempo imersão & 111 & 146 & 183 \\
\hline $0,6 \%$ & $36 \mathrm{~s}$ & $1,08 \mathrm{a}$ & $0,22 \mathrm{~b}$ & $0,58 \mathrm{~b}$ \\
$0,6 \%$ & $84 \mathrm{~s}$ & $1,20 \mathrm{a}$ & $0,42 \mathrm{~b}$ & $0,58 \mathrm{~b}$ \\
$1,0 \%$ & $60 \mathrm{~s}$ & $1,43 \mathrm{a}$ & $0,27 \mathrm{~b}$ & $0,72 \mathrm{~b}$ \\
\hline $0,1 \%$ & $36 \mathrm{~s}$ & $1,39 \mathrm{a}$ & $0,26 \mathrm{~b}$ & $0,64 \mathrm{~b}$ \\
$0,6 \%$ & $6 \mathrm{~s}$ & $1,14 \mathrm{a}$ & $0,22 \mathrm{~b}$ & $0,67 \mathrm{~b}$ \\
$0,0 \%$ & $0 \mathrm{~s}$ & $2,28 \mathrm{a}$ & $0,39 \mathrm{~b}$ & $0,77 \mathrm{~b}$ \\
\hline C.V. $(\%)$ & & 36,80 &
\end{tabular}

$\left({ }^{1}\right)$ Doses de óleo de eucalipto, em porcentagem na solução/tempo de imersão das mudas, em segundos.

Médias seguidas pelas mesmas letras nas linhas não diferem entre si, pelo teste de Scott-Knott, a 5\% de probabilidade.

ocorreu no início de dezembro, quando as temperaturas variaram entre a mínima de $20^{\circ} \mathrm{C}$ e a máxima de $33^{\circ} \mathrm{C}$.

Nas condições em que foi instalado o experimento concluiu-se que, para se obter maior produção de raízes comerciais da mandioquinha-salsa, a partir de mudas pré-enraizadas em bandejas, deve-se desinfectar as mudas em solução aquosa com doses de $0,6 \%$ ou $1,0 \%$ de óleo de eucalipto e imersão em tempos de $36 \mathrm{~s}$ a $84 \mathrm{~s}$, sendo a colheita aos 264 dias após o plantio.

\section{AGRADECIMENTOS}

Ao CNPq, pelas bolsas concedidas; à FUNDECTMS, pelo apoio financeiro e à UEM, pela oportunidade para efetuar o Pós-doutorado.

\section{REFERÊNCIAS}

BRUNE, S.; GIORDANO, L.B.; LOPES, C.A.; MELO, P.E. Tratamento químico de mudas de mandioquinha-salsa. Horticultura Brasileira, v.14, p.207-210, 1996.

HEREDIA ZÁRATE, N.A.; VIEIRA, M.C.; GRACIANO, J.D.; FIGUEIREDO, P.G.; BLANS, N.B.; CURIONI, B.M. Produtividade de mandioquinha-salsa sob diferentes densidades de plantio e tamanho das mudas. Ciência e Agrotecnologia, v.33, p.139-143, 2009.

HEREDIAZÁRATE, N.A.; VIEIRA, M.C.; RECH, J.; GRACIANO, J.D.; GOMES, H.E.; PONTIM, B.C.A. Número de fileiras no canteiro e espaçamento entre plantas na produção e na rentabilidade da beterraba em Dourados, Estado do Mato Grosso do Sul. Acta Scientiarum: Agronomy, v.30, p.397-401, 2008a.

HEREDIA ZÁRATE, N.A.; VIEIRA, M.C.; RECH, J.; QUAST, A.; PONTIM, B.C.A.; GASSI, R.P. Yield and gross income of arracacha in monocrop and intercropping with the Japanese bunching onion and parsley. Horticultura Brasileira, v.26, p.277-281, 2008b.

KUHN, O.J. Indução de resistência em feijoeiro (Phaseolus vulgaris) por acinbezolar-S-metil e Bacillus cereus: aspectos fisiológicos, bioquímicos e parâmetros de crescimento e produção. 2007. 138 f. Tese (Doutorado em Agronomia). Universidade de São Paulo, Piracicaba, 2007.

LEBLANC, R.E.G.; PUIATTI, M.; SEDIYAMA, M.A.N.; FINGER, F.L.; MIRANDA, G.V. Influência do préenraizamento e de tipos de mudas sobre a população, crescimento e produção da mandioquinha-salsa "Roxa de Viçosa". Revista Ceres, v.55, p.74-82, 2008.

MADEIRA, N.R.; SOUZA, R.J. Mandioquinha-salsa: alternativa para o pequeno produtor. Disponível em http: / / www.editora. ufla.br/BolTecnico/pdf/bol_60.pdf. Acesso em 18-3-2008.

SCHWAN-ESTRADA, K.R.F.; STANGARLIN, J.R.; CRUZ, M.E.S. Uso de extratos vegetais no controle de fungos fitopatogênicos. Floresta, v.30, p.129-138, 2000.

TURRENT, A.; LAIRD, R.J. La matriz experimental Plan Puebla, para ensayos sobre prácticas de producción de cultivos. Agrociencia, v.19, p.117-143, 1975. 
